\title{
A Case Survived Three Cancers Helped by Chinese Medicine
}

\author{
Dan Jiang ${ }^{1-3 *}$, Fanyi Meng ${ }^{4}$ and Jenny Meng ${ }^{5}$ \\ ${ }^{1}$ Principal of Hallam Institute of TCM, UK \\ ${ }^{2}$ Vice-principal of Asante Academy of TCM affiliated with Middlesex University, UK \\ ${ }^{3}$ Clinical TCM Consultant to GP-TCM Research Association, and Visiting professor of Beijing University of Chinese Medicine, China \\ ${ }^{4}$ Programme Leader/Senior Lecturer, BSc(Hons) Acupuncture, Lincoln College, UK \\ ${ }^{5}$ Dementia Practitioner, Frailty Assessment Unit, Lincoln County Hospital, UK
}

*Corresponding author: Dan Jiang, Principal of Hallam Institute of TCM, UK, E-mail: djiang52@hotmail.com

Received: 18 Feb, 2019 | Accepted: 14 Mar, 2019 | Published: 19 Mar, 2019

Citation: Jiang D, Meng F, Meng J (2019) A Case Survived Three Cancers Helped by Chinese Medicine. J Clin Case Stu 4(1): dx.doi.org/10.16966/24714925.185

Copyright: (C) 2019 Jiang D, et al. This is an open-access article distributed under the terms of the Creative Commons Attribution License, which permits unrestricted use, distribution, and reproduction in any medium, provided the original author and source are credited.

Keywords: Chinese Medicine (TCM); Herbal medicine; Acupuncture; Cancers; Case report

\section{Introduction}

Cancer is a primary healthcare concern with high mortality rate worldwide. Although the advance of medical procedures and new ways of treating cancer developed quickly in the past decade, amid of genetic and immune-therapies, it remains the second global leading cause of death [1]. Because of the difficulty in treating cancers, Chinese medicine has been introduced in clinical practice along with the mainstream medical treatment broadly in China and many Eastern Asia countries, and has been accepted as a useful complementary for those suffered the side effects of chemotherapy and radiotherapy. Chinese herbal medicine is the most applied modality of such practice and varies aspects, from pain, nausea, anemia, damage in immune function to hair loss are tested $[2,3]$. Nearly all researches measuring Quality of Life (QoL) in Chinese medicine on cancer agreed that Chinese herbal medicine improves QoL in cancer patients suffering from side effects of chemo and radio therapies [4]. Acupuncture is obviously an indispensable part of the practice, and is particularly common out of China [5]. Moxibustion is also investigated for its benefits [6].

However, the treatment of cancers themselves by Chinese medicine therapies are still in early stage, although numerous laboratory tests proved some Chinese herbs are effective in killing cancer cells and suppressing tumour growth, it is hardly any broad consensus being reached in supporting using Chinese medicine to treat cancer alone. The advantage of using Chinese herbs with conventional medicine therapies together is considered to presenting real benefit, including enhance the chemotherapy completion rate, maintaining immune function at good level. Therefore, Chinese medicine should be used as an adjunctive therapy along with mainstream medicine $[7,8]$.

The authors have been practicing Chinese medicine form more than 30 years, and many patients came to the clinic have experienced cancer related illness. Most of them are well controlled and in the remission stage, actually, the treatments provided are for common conditions including migraine, digestive problems (for example, IBS), insomnia and depressions. Some of them are still receiving regular chemotherapy and other cancer treatment, but need helps for the fatigue, general aching, loss of hair, anaemia, etc. Usually, when patients came for Chinese medicine treatment for cancer as the main treatment, they are redirected to appropriate medical authorities following the professional body's guideline.

In some cases, the patients are in late stages with no hope for miracles and gave up their medical treatment. It was clear that during the rest of their time, they are very clear about the consequence of not receiving the recommended treatment. What they wanted was the relatively less painful life. When full explanation and double checked consent was given, Chinese medicine treatments were provided, and advice of resuming regular medical treatment was always given to them.

One of the cases treated in Jiang's clinic (the first author's clinic) has raised interest among Chinese medicine circle and also the medical professionals who are in charge of her medical treatment. Therefore, the authors consider the report of such a case might provide a clue of integrating Chinese medicine within the treatment/management of cancers.

\section{Patient Information}

Ms J B (pseudo name for the convenient of discussion), 37 years on first visit, 56 years now.

First visit: May 2000

Episode One: Chief complaints: seeking help for suffering from breast cancer stage III.

J was referred to Jiang by a local TCM practitioner. She found a lump at the upper lateral region of her left breast and the lymph nodes under of left armpit had been involved roughly 8 month ago. After visiting her GP, she was initially diagnosed as breast cancer in the 
III stage and the diagnosis was confirmed by MRI and other relevant tests. An operation was arranged immediately for removing the local cancer tissue and affected lymph nodes. The surgical procedure was considered to be satisfactory, and she was given a half year course of chemotherapy afterwards. At the time of first visit, she was discharged only two weeks after all treatments being completed.

She manifested fatigue, anxiety, depression, a normal scar in left breast. The lesion at armpit healed and recovered well. But hard tissues are broad felt in the left breast. Two lumps were palpated and movable in the right breast which was sized $1-2 \mathrm{~cm}$ with mild pain when pressed. Chinese medicine tongue observation reported light red tongue with less white coating. The pulse was wiry and thin.

Syndrome Pattern established with TCM: Liver Qi stagnation and Spleen deficiency; Blood stasis.

\section{TCM treatment}

Acupuncture: Du 20 Baihui, Du 24 Shenting, Li 14 Binao, Li 11 Quchi, Sj 5 Waiguan, Gb 41 Zulinqi, St 36 Zusanli, Sp 9 Yinlingquan, Sp 6 Sanyinjiao

Fine needles are used to the acupuncture points, with moderate stimulation.

Herbal prescription: (all dosages are in grams)

Banzhilian (Herba Scutellariae Barbatae)-15

Huangqi (Radix Astragali Membranacei)-15

Danggui (Radix Angelicae Sinensis)-10

Dangshen (Radix Codonopsis Pilosulae)-10

Chaihu (Radix Bupleuri)-10

Xiakucao (Spica Prunellae Vulgaris)-10

Huzhang (Rhizoma Polygonicuspidati)-10

Ruxiang (Gummi Olibanum)-10

Chenpi (Pericarpium Citri Reticulatae)-10

Chishao (Radix Paeoniae Rubrae)-10

Baishao (Radix Paeoniae Lactiflorae)-10

Zhigancao (Radix Glycyrrhizae Uralensis)-5

One dose per day, 4 dose per week.

The daily dose of herbs was simmered as decoction, orally administrated twice daily. A week after, during her $2^{\text {nd }}$ visit, the same acupuncture was given, and the herbal prescription was repeated.

Since her response was very positive. Then in the following the above routine of acupuncture and herbal medicine treatment maintained for a year.

Her conditions keep improving steadily. At the end point of the year, she felt fully recovered. All lumps in the breast had disappeared. The mind was fit and energy returned to normal level (the level of before the discovery of the cancer). The treatment completed.

\section{Episode Two}

The tumours in the liver (Hepatocellular Carcinoma-HCC).

In the end of 2006, the patient revisited Jiang's clinic again because she was found that multiple tumours of $2-6 \mathrm{~cm}$ various size in her liver. She was done a biopsy from liver tissue for confirming whether occurred malignant or not? But unfortunately, the tumours were diagnosed as primary hepatocellular carcinoma (HCC). But luckily, there was no sign of migration to other important organs. Because the tumours sit around of portal vein of liver, it might be dangerous to remove them with surgical operation; accordingly oncological surgeon didn't plan an operation immediately. She was also told that chemotherapy was also less promising in her case. Based on her treatment experience and the overall balance of chemo and radio therapy and their potential damage, she decided to seek advice and treatment from Jiang's clinic for help.

She manifested the pain in right costal area, upper abdominal distension, loose bowel, anxiety and insomnia. The situation was discussed and the potential complications well explained. Based on the previous research and personal experience, Jiang offered treatment aimed at keeping the overall health in good condition, hoping the cancer might be controlled. Consent was given.

Syndrome Patterns of TCM: Liver Qi stagnation and Blood stasis.

\section{TCM treatment}

Acupuncture: Du 20 Baihui, Du 24 Shenting, Ren 13 Shangwan, Ren 12 Zhongwan, St 25 Tianshu, Sp 9 Yinlingquan, Gb 34 Yanglingquan, Sj 5 Waiguan, Gb 41 Zulinqi, Li 4 Hegu, Liv 3 Taichong

Herbal medicine: Kang Ni Ling capsules (Ginseng, Lingzhi, Dangshen, Ciwujia and Sanqi, etc)

Chai hushugan Wan/Jiaweixiaoyao Wan

Ganoderma Spore Powder (External plaster of Anti-cancer purpose)

The first and second were for oral administration. They were ready made TCM pills. The dosages were following the instructions. The third one was external Chinese medical plaster, applying on the skin of liver area daily. The ready-made herbal remedies are sometimes replaced with Chinese herbal decoction with prescription as following:

Huangqi (Radix Astragali Membranacei)-30
Sharen (Fructus Amomi)-10
Chenpi (Pericarpium Citri Reticulatae)-10
Fuling (Sclerotium Poriae Cocos)-10
Xiangfu (Rhizoma Cyperi Rotundi)-10
Shanyao (Radix Dioscoreaeoppositae)-10
Baizhu (RhizomaAtractylodis Macrocephalae)-10
Huzhang (Rhizoma Polygonicuspidati)-10
Xiakucao (Spica Prunellae Vulgaris)-10
Chaihu (Radix Bupleuri)-10
Tufuling (Rhizoma Smilacis Glabrae)-10
Zhigancao (Radix Glycyrrhizae Uralensis)-5

The patient visited Jiang clinic weekly or biweekly for three years with the above summarized treatment plans. She appeared in a good life quality and moderate mind without any pain at the liver region, but the tumors still existed. As the overall situation was evaluated again, the patient was advised to seek opinion on if she can go for a surgical removal of those tumours.

The complication from liver operation: In 2009, a consultant called for a multi-agency meeting on her case. The conclusion was that the situation remained unchanged, and the diagnosis standing (HCC). Therefore, the recommendation is there is no better chance in conventional therapy. However the patient insisted for a radical consideration. 
In September 2009, she was accepted by a consultant Hepatobiliary \& Laparoscopic surgeon in the hospital of another city for removing the tumours in the liver, together with a microwave ablation to several of the remaining tumours in her liver. During the surgical operation, histology of large lesion was done and proved to be a neuroendocrine tumour. And no malignant tissue was found in her liver.

The surgical operation was a great success. She got her mind relieved. However, the operation brought with a postoperative infected hematoma which had to be drained. Since there were two draining tubes, one of them was drained from the bottom of right lung, and another one was drained from the liver; from both of these two tubes, she was drained more than $100 \mathrm{ml}$ liquid out daily; so liquid accumulated in abdominal cavity and ascites was the trouble.

She experienced bad swollen on both of her legs and abdominal pain/distension. Due to struggling with bad pain around draining openings, she couldn't lied at on the bad flat, having to leaning at the left side with a half sitting position. She was exhausted physically and mentally, and loses weight. Other symptoms include poor appetite, anxiety, stress, depression and insomnia. Constipation developed due to taking Morphine etc stronger painkillers.

The situation was desperate, and she came back to Jiang's clinic for help four months after the operation.

Clinical observation: She was struggling to move and kept at a compulsive position who had to leaning at left side of setting, two draining openings with many yellow discharge from bottom of right lung and liver; pressed abdominal pain, distension with moveable sound in the abdomen; oedema at both of legs from knees to ankles.

TCM tongue observation: Swollen and light red tongue with white coating.

The pulse is wiry.

Impression of conventional medical diagnosis:

$\alpha$ Ascites and oedema secondary to portal hypertension and wound infection

\section{B) Hypoproteinaemia}

\section{Syndrome pattern of TCM}

Qi and yang deficiency of spleen and kidney; excessive dampness and internal fluid accumulation.

Treating principle: To eliminate excessive fluid from the three burners; to warm Yang and to reinforce Qi

As the patient could not lied supine in a relaxed manner due to the position of her drains, acupuncture could not be used. She therefore received only herbal medicine.

\footnotetext{
Herbal medicine prescription

Huangqi (Radix Astragali Membranacei)-30

Danggui (Radix Angelicae Sinensis)-10

Yiyiren (Semen Coicis Lachyrma-jobi)-30

Fuling (Sclerotium Poriae Cocos)-10

Guizhi (Ramulus Cinnamomi Cassiae)-10

Tinglizi (Semen Descurainiae SeuLepidii)-10

Houpo (Cortex Magnoliae Officinalis)-10

Zhishi (Fructus Immaturus Citri Aurantii)-10

Shanzha (Fructus Crataegi)-10
}

Sharen (Fructus Amomi)-10

Shenqu (Massa Medica Fermentata)-10

Zhigancao (Radix Glycyrrhizae)-5

(Taken twice a day as a juice)

Renshen (ginseng) extract juice was also prescribed twice daily.

After taking above herbs for two weeks, her draining fluid was reduced $20 \mathrm{ml}$ daily. The same prescription was repeated until doctors took one draining tube out from lung; another tube out in one month. The ascites and oedema were both reduced significantly, and could lie at flat.

It took another 4 months to have her fully rehabilitated from the operation and subsequence.

\section{Episode Three}

In the end of 2014, the patient found a lump of hard texture in her left breast. Breast cancer was diagnosed soon. It was believed to be a new one at stage I. Chemotherapy was given first for three months. Then the tumours was smaller that allowed the surgical removal of the tumour. And a mastectomy was done as a radical treatment.

Learnt from previous experience, she had been using TCM treatment throughout her medical treatment. According to her account, she recovered quickly from the chemotherapy each time. She actually did not experience the side effect of nausea, hear loss and exhaustion. The treatments she received were in line with the episode one, consisting of acupuncture weekly and herbal decoction weekly with similar prescriptions.

Since the recovery from mastectomy, she has been back to a parttime work and living a normal life.

Since she started her menopause, she visits the clinic regular again for anxiety and possibly depression. That enables the author to have a thorough review of the case with long following up.

Summary of the case: The patient suffered from breast cancer twice and also a liver tumour of neuro-endocrine origin. The first and second episodes are both discussed in the subject of cancer. The conventional medical therapies were given to her as the main treatment. However, each time, the damage caused to her body was huge, and she eventually turned to TCM for recovery. The treatment of acupuncture and Chinese herbal medicine was lengthy, still, both the patient and the practitioner hold up and achieved fabulous outcome. Full recovery was achieved twice.

The third time, she actively seek help from TCM, from the beginning of the conventional treatment. This allowed her a relative easier treatment course, and the completion was satisfactory. She is now 57 and living in an otherwise healthy and working life.

\section{Discussion and Conclusion}

The rule of TCM in her case was always in supporting her body's own recovery and repairing mechanism. In TCM terms, the strengthening of Qi and Blood, who supply the energy and material for rebuild the body. In the prescriptions, Huang Qi and Dang Gui were always used as chiefs for the purpose.

The combination of Huang Qi and Dang Gui forms a famous formula called Dang Gui Bu Xue Tang, which aimed to generate blood from Qi. It was created by LI Dong Yuan for the recovery from high fever, trauma, infections, and child birth with features of lingering weakness [9]. 
Huangqi (Astragali) has been proven to be extremely useful in cancer patient supporting treatment [10]. It is clinical observed to be able to adjust the immune function, leading to inhibiting tumour growth, and protecting the damage from chemo and radio therapies. Danggui (angelica) is even more recognised in helping cancer survival $[11,12]$. The combination of the two has been demonstrated to be more effective in promoting blood ingredients particularly in the case of renal anaemia, and cancer therapies related anaemia [13].

From the experience of the authors, those two should always been used in the case when patients are suffering from lower immune function, anaemia, and general weakness. And all reports available suggest they are of very low risk of any side effect.

The maintaining of good level of energy, good immune function are essential for patients to maintain a good life, and they are the two key elements of all Quality of Life (QoL ) measurements.

On the other hand, the stagnation of liver Qi, as a pattern, is a common theme of cancer patients. The pattern is closely related to emotional trauma and stress. According to Burgess, half of breast cancer patients are suffering from anxiety and depression [14]. They play a detreating role in making the life more difficult and the affecting the overall QoL. "Depression affects up to $20 \%$, and anxiety $10 \%$, of patients with cancer, compared with figures of $5 \%$ and $7 \%$ for past-year prevalence in the general population" $[15,16]$. The overall situation is not getting better, after this issue was raised 20 years ago. From clinical studies, most of anxiety and depression cases belong to the pattern of Liver Qi Stagnation (LQS).

TCM believers that Liver Qi move along breast and govern the motion; if Liver Qi unsmooth moves in the breasts where there are such more scar on these to cause a bad emotional symptoms. Regular acupuncture and some patent herbs can control her symptoms and protect her keeping in a happy life.

To address the anxiety and stress is actually as important as the strengthening of Qi and blood. If the liver qi and moving free of trouble, the body will have a much better chance of repairing and recovering.

The importance of patient positive attitude and the therapeutic bond. The author recognised that the fact of the patient stay with TCM treatment from 6 months to 3 years is a manifesto of the trust in TCM and the practitioner. Only when the patient is in fully cooperative therapeutic relationship, this is made possible.

This firm relationship can benefit the patient in the following ways: the good treatment plan is stick on for enough time to be tested; the mental and emotional support from the trusted practitioner is of huge boost to the morale of the patient; and the regular visiting makes the patient in good social connection with outside world.

In conclusion, the authors believe this is an interesting case demonstrated how TCM can play a supportive role in cancer patient management. With such a difficult case of two cancer episodes and a destructive tumour disaster, she recovered and cleared of all cancer tumours, and lives an otherwise normal life. The value and principle might provide a clue to future practice.

\section{References}

1. World Health Organization (WHO) (2018) Cancer: Key Fact World Health Organization: Geneva.

2. Lau CH, Wu X, Chung VC, Liu X, Hui EP, et al. (2016) Acupuncture and Related Therapies for Symptom Management in Palliative Cancer Care: Systematic Review and Meta-Analysis. Medicine (Baltimore) 95: e2901.

3. Jiang D, Meng FY, Li L, Qu F (2016) The Role of Chinese Medicine in Cancer Care-a Critical Review. World J Tradit Chin Med 2: 68-73.

4. Chen X, Deng L, Jiang X, Wu T (2016) Chinese medicinal herbs for oesophageal cancer. Cochrane Database of Syst Rev.

5. Paley CA, Johnson MI, Tashani OA, Bagnall AM (2015) Acupuncture for cancer pain in adults. Cochrane Database Syst Rev.

6. Zhang H, Lin Z, Cheung F, Cho W, Tang J (2018) Moxibustion for alleviating side effects of chemotherapy or radiotherapy in people with cancer. Cochrane Database of Syst Rev 11.

7. Ling CQ, Yue XQ, Ling C (2014) Three advantages of using traditional Chinese medicine to prevent and treat tumor. J Integr Med 12: 331335.

8. Qi F, Zhao L, Zhou A, Zhang B, Li A, et al. (2015) The advantages of using traditional Chinese medicine as an adjunctive therapy in the whole course of cancer treatment instead of only terminal stage of cancer. Biosci Trends 9: 16-34.

9. Deng Z (2011) Formulary of Chinese Herbal Medicine. Chinese TCM Publishing Hourse, Beijing.

10. Jung $\mathrm{Y}$, Jerng $\mathrm{U}$, Lee $\mathrm{S}$ (2016) A systematic review of anticancer effects of Radix Astragali. Chin J Integr Med 22: 225-236.

11. Liao KF, Chiu TL, Huang SY, Hsieh TF, Chang SF, et al. (2018) Anti-Cancer Effects of Radix Angelica Sinensis (Danggui) and $\mathrm{N}$-Butylidenephthalide on Gastric Cancer: Implications for REDD1 Activation and mTOR Inhibition. Cell Physiol Biochem 48: 22312246.

12. Reddy CS, Kim SC, Hur M, Kim YB, Park CG, et al. (2017) Natural Korean Medicine Dang-Gui: Biosynthesis, Effective Extraction and Formulations of Major Active Pyranocoumarins, Their Molecular Action Mechanism in Cancer, and Other Biological Activities. Molecules 22.

13. Zhang K, Chen Q, Wang X, YiZ, Zhang B (2013) The Protective Effective on Bone Marrow of Dang Gui Bu Xue Tang in Chemotherapy. Med Res Education 30: 93-96.

14. Burgess C, Cornelius V, Love S, Graham J, Richards M, et al. (2005) Depression and anxiety in women with early breast cancer: five year observational cohort study. BMJ 330: 702.

15. Pitman A, Suleman S, Hyde N, Hodgkiss A (2018) Depression and anxiety in patients with cancer. BMJ 361: k1415.

16. Wang WL, Sheu SY, Chen YS, Kao ST, Fu YT, et al. (2015) Enhanced Bone Tissue Regeneration by Porous Gelatin Composites Loaded with the Chinese Herbal Decoction Danggui Buxue Tang. PLoS One 10: e0131999. 